\title{
Magnetic Field Effect on Multiplicity of Solutions Induced by Thermosolutal Convection in a Bénard Square Porous Cavity Submitted to Horizontal Concentration Gradient
}

\author{
A. Mansour $\dagger$, M. Ait Ahmed, A. Amahmid, M. Hasnaoui, I. Filahi and Y. Dahani \\ Cadi Ayyad University, Faculty of Sciences Semlalia, Department of Physics, \\ LMFE, BP 2390 Marrakesh, Morocco \\ $†$ †orresponding Author Email: a.mansour@uca.ma
}

(Received October 14, 2020; accepted March 2, 2021)

\begin{abstract}
In this work, we present a numerical study of the magnetic field effect on double diffusive natural convection in a square porous cavity saturated with an electrically conducting binary mixture. The cavity is heated from below and cooled from the top, while its vertical walls are adiabatic and maintained at constant but different concentrations. The numerical results are obtained for a Lewis number $L e=10$ and the following ranges for the other controlling parameters: $40 \leq R_{T} \leq 1000,-0.2 \leq N \leq 0.2$ and $-0.5 \leq H a \leq 0.5$, where $R_{T}, N$ and $H a$ are the thermal Rayleigh number, the buoyancy ratio and the Hartmann parameter, respectively. First, the effect of the Hartmann parameter on the maintenance and disappearance of the multiple steady state solutions obtained in the case of purely thermal convection is examined. Then, the combined effect of $N$ and $\mathrm{Ha}$ on the existence of these steady solutions is analyzed. It is found that the critical values of $N$ corresponding to the transitions between the different solutions are modified by the application of a magnetic field. However, the nature of the transitions is unchanged. It is shown that the magnetic field may affect considerably the flow intensity and the heat and mass transfer in the medium.
\end{abstract}

Keywords: Numerical study; Porous media; Rayleigh-Benard flow; Natural convection; Heat and mass transfer; Magnetic field.

\section{NOMENCLATURE}

$B_{0} \quad$ strength of the magnetic field

BF Bicellular Flow

BAF Bicellular Anti-natural Flow

BNF Bicellular Natural Flow

$D \quad$ mass diffusivity

$\mathrm{Ha}$ Hartmann number

$g$ gravitational acceleration

$K \quad$ permeability of the porous medium

$L^{\prime} \quad$ length of the porous cavity

Le Lewis number

MF Monocellular Flow

MCF Monocellular Clockwise Flow

MTF Monocellular Trigonometric Flow

$N$ buoyancy ratio

$\mathrm{Nu} \quad$ Nusselt number

$R_{T} \quad$ thermal Rayleigh number

$S$ dimensionless concentration

$S_{0}^{\prime} \quad$ dimensional concentration of the left wall

$S_{1}^{\prime}$ dimensional concentration of the right wall

Sh Sherwood number

$\Delta S^{\prime} \quad$ concentration difference
$T_{c}^{\prime} \quad$ dimensional temperature at the upper

horizontal wall of the cavity

$T_{h}^{\prime} \quad$ dimensional temperature at the lower horizontal wall of the cavity

$\Delta T^{\prime} \quad$ temperature difference

$(u, v)$ dimensionless velocities in $(x, y)$ directions

$(x, y)$ dimensionless coordinates

$\alpha \quad$ thermal diffusivity

$\beta_{S} \quad$ solutal expansion coefficient

$\beta_{T} \quad$ thermal expansion coefficient

$\varepsilon \quad$ normalized porosity

$\varepsilon^{\prime} \quad$ porosity of the porous medium

$\lambda$ thermal conductivity of the saturated porous medium

$v \quad$ kinematic viscosity of the fluid

$\mu \quad$ dynamic viscosity of the fluid

$\epsilon \quad$ electrical conductivity of the fluid

$\rho \quad$ density of the fluid mixture

$(\rho c)_{f}$ heat capacity of the fluid mixture

$(\rho c)_{p}$ heat capacity of the saturated porous 


$\begin{array}{ll}t & \text { dimensionless time } \\ \mathrm{T} & \text { dimensionless temperature } \\ \mathrm{TCF} & \text { tricellular flow with a clockwise central } \\ & \text { cell } \\ \mathrm{TF} & \text { tricellular flow } \\ \mathrm{TTF} & \text { tricellular flow with a trigonometric } \\ & \text { central cell }\end{array}$

\section{INTRODUCTION}

The magnetic field is a good control parameter on heat transfer and fluid flow and is also used to reach maximum efficiency in thermodynamics in different fields (Kabeel et al. 2015; Kasaeian et al. 2017). The problem of natural convection in an electrically conducting fluid under a magnetic field has been the subject of numerous studies (Hussein et al. 2014; Bahiraei and Hangi 2015). This interest stems from the implication of the phenomenon in many engineering applications such as crystal growth processes.

Several researchers have studied the effect of the magnetic field on pure thermal convection. Here we cannot quote all these works but we will present some investigations. In fact, the influence of a uniform magnetic field on natural convection in square cavity was studied by Krakov and Nikiforov (2002). They discovered that the angle between the direction of the temperature gradient and magnetic field influences the convective structure and the heat flux. Sophy et al. (2005) studied the thermomagnetic convection in a differentially heated square cavity. These authors showed that the flow pattern underwent a great modification when the maximum value of the magnetic field was beyond a critical threshold and heat exchange at the walls increases. Zeng et al. (2007) studied numerically natural convection in an enclosure filled with a fluid-saturated porous medium and submitted to a strong magnetic field. Two physical configurations were considered by these authors. The first one was heated from the bottom and cooled from the top, and the second was heated from the left side vertical wall and cooled from the opposite wall. An electric coil was set below this enclosure to generate a magnetic field. A numerical investigation for penetrative ferroconvection via internal heat generation in a ferrofluid saturated porous layer was performed by Nanjundappa et al. (2012). Heidary et al. (2016) analyzed natural convection in porous inclined enclosures, equipped with one or two obstacles, with the presence of sinusoidal heated wall and magnetic field. Hoshyar et al. (2016) showed that the Least Square Method (LSM) is a powerful and easy-to-use analytic tool for predicting the temperature distribution in a porous fin which was exposed to uniform magnetic field. An experimental study of heat transfer enhancement due to laminar ferrofluid flow in a horizontal tube medium

heat capacity ratio

dimensionless stream function

\section{Subscripts \\ cr critical value \\ ext extremum value \\ max maximum value \\ Superscripts}

dimensional variable

partially filled with porous media under fixed parallel magnet bars was carried out by Sheikhnejad et al. (2017). In this study, it was found that the presence of both porous media and magnetic field simultaneously could highly improve heat transfer. The impact of an external magnetic field on the hydrothermal aspects of natural convection of a power-law non-Newtonian nanofluid inside a baffled U-shaped enclosure was examined by Ali et al. (2020). They reported that the cooling performance of a cavity augments with the rise of aspect ratio, nanoparticle volume fraction, Rayleigh number, while it is reduced by boosting the Hartmann number.

On another side, many efforts have been devoted by the researchers to understand the effect of magnetic field on double diffusion. In this frame, Chamkha and Al-Naser (2002) studied numerically the characteristics of hydromagnetic double-diffusive convective flow of a binary gas mixture in a rectangular enclosure with the upper and lower walls being insulated, while constant temperatures and concentrations are imposed along the left and right walls. A uniform magnetic field was applied in the $\mathrm{x}$ direction. The same authors conducted a similar study by imposing heat and mass fluxes on the vertical walls (2002). Robillard et al. (2006) studied analytically and numerically the electromagnetic field effect on the natural convection in a vertical porous cavity saturated with an electrically conducting binary mixture. An analytical and numerical investigation was conducted by Ramambason and Vasseur (2007) to study the effect of an electromagnetic filed on natural convection in a horizontal shallow porous cavity filled with an electrically conducting binary mixture. A uniform heat flux was applied on the horizontal walls of the layer while the vertical walls were adiabatic. Numerical simulation of double-diffusive natural convective flow in an inclined rectangular enclosure in the presence of magnetic field and heat source was conducted by Teamah et al. (2012). The effect of magnetic field on 3D double diffusive convection in a cubic cavity filled with a binary mixture was considered by Maatki et al. (2013). The influence of the magnetic field on the structure of the threedimensional flow, the distribution of temperature and concentration and the different characteristics of heat and mass transfer of the thermal and solutal dominated region were presented. Teamah et al. (2016) investigated the magnetic field effect on double diffusive convection in a trapezoidal enclosure. 
In this investigation, the attention is focused on the magnetic field effect on the multiple steady state solutions induced in a square porous cavity saturated with an electrically conducting binary mixture. The cavity is heated from below and cooled from the top, while its vertical walls are adiabatic and maintained at constant but different concentrations. This configuration was considered in the past by Altawalbeh et al. (2013) without taking into account the multiplicity of solutions generated for adequate ranges of the governing parameters. Our objective in this work is to examine the influence of the magnetic field on the existence range of different types of steady-state solutions. Results for heat and mass transfer induced by these solutions are also presented and discussed. Note that in previous studies conducted by Robillad et al. (1998), Bourich et al. (2004) and Mansour et al. (2006), it was shown that these quantities may be considerably affected by the type of solution.

\section{MATHEMATICAL FORMULATION}

The system under consideration (Fig. 1) is a square cavity filled with an isotropic and homogenous porous medium, saturated with an electrically conducting binary mixture. The bottom wall of the cavity is maintained at a hot temperature $T_{h}^{\prime}$, while the top one is maintained at a cold temperature $T_{c}^{\prime}$. The right vertical wall is maintained at a concentration $\left(S_{1}^{\prime}\right)$ higher than that of the left one $\left(S_{0}^{\prime}\right)$. The top and bottom walls are assumed impermeable to mass transfer, while the right and left ones are assumed adiabatic. A magnetic field of strength $B_{0}$ is applied in a direction normal to the horizontal walls of the porous cavity. The thermophysical properties of the binary fluid are considered constant except the density in the buoyancy term which varies linearly with the local temperature and concentration (Boussinesq approximation):

$$
\rho=\rho_{0}\left[1-\beta_{T}\left(T^{\prime}-T_{0}^{\prime}\right)-\beta_{S}\left(S^{\prime}-S_{0}^{\prime}\right)\right]
$$

where $\beta_{T}$ and $\beta_{S}$ are, respectively, the thermal and solutal expansion coefficients of the binary fluid, $T$, is the dimensional temperature and $S^{\prime}$ is the concentration ( $T_{0}^{\prime}$ and $S_{0}^{\prime}$ correspond to the reference state). Viscous dissipation is neglected and the solid matrix and the fluid are assumed to be at local thermal equilibrium. Using the Darcy model and taking into account the magnetic field effect, the dimensionless equations governing the flow and heat and mass transfer in the saturated porous medium are written as follows:

$$
\begin{aligned}
& \frac{\partial^{2} \boldsymbol{\Psi}}{\partial^{2} \boldsymbol{X}}+\left(1+\boldsymbol{H a}^{2}\right) \frac{\partial^{2} \boldsymbol{\Psi}}{\partial^{2} \boldsymbol{Y}}=-\boldsymbol{R}_{\boldsymbol{T}}\left(\frac{\partial \boldsymbol{T}}{\partial \boldsymbol{X}}+\boldsymbol{N} \frac{\partial \boldsymbol{S}}{\partial \boldsymbol{X}}\right) \\
& \frac{\partial \boldsymbol{T}}{\partial \boldsymbol{t}}+\boldsymbol{U} \frac{\partial \boldsymbol{T}}{\partial \boldsymbol{X}}+\boldsymbol{V} \frac{\partial \boldsymbol{T}}{\partial \boldsymbol{Y}}=\nabla^{2} \boldsymbol{T} \\
& \boldsymbol{\varepsilon} \frac{\partial \boldsymbol{S}}{\partial \boldsymbol{t}}+\boldsymbol{U} \frac{\partial \boldsymbol{S}}{\partial \boldsymbol{X}}+\boldsymbol{V} \frac{\partial \boldsymbol{S}}{\partial \boldsymbol{Y}}=\frac{1}{\boldsymbol{L} \boldsymbol{e}} \nabla^{2} \boldsymbol{S}
\end{aligned}
$$

$\mathrm{U}=\frac{\partial \Psi}{\partial \mathrm{Y}} \quad$ and $\quad \mathrm{V}=-\frac{\partial \Psi}{\partial \mathrm{X}}$

where $\Psi, T$, and $S$ are the dimensionless stream function, temperature and concentration, respectively. $U$ and $V(X$ and $Y$ ) are the dimensionless horizontal and vertical velocities (coordinates).

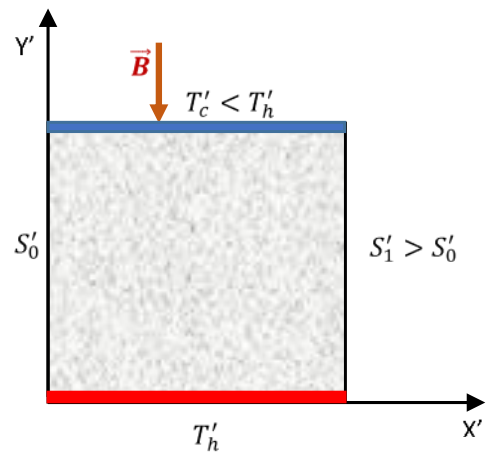

Fig. 1. Schematic diagram of the physical problem.

The hydrodynamic, thermal and solutal boundary conditions associated with the present problem are:

$$
\left.\begin{array}{l}
\text { For } X=0 \text { and } 0 \leq Y \leq 1: \Psi=0, \frac{\partial T}{\partial X}=0, S=0 \\
\text { For } X=1 \text { and } 0 \leq Y \leq 1: \Psi=0, \frac{\partial T}{\partial X}=0, S=1 \\
\text { For } Y=0 \text { and } 0 \leq X \leq 1: \Psi=0, \frac{\partial S}{\partial Y}=0, T=1 \\
\text { For } Y=1 \text { and } 0 \leq X \leq 1: \Psi=0, \frac{\partial S}{\partial Y}=0, T=0
\end{array}\right\}
$$

The above dimensionless equations show that the solutions for the present configuration are governed by five dimensionless parameters, namely, the thermal Rayleigh number $R_{T}$, the buoyancy ratio $N$, the Lewis number $L e$, the Hartmann number $\mathrm{Ha}$ and the normalized porosity $\varepsilon$. They are respectively defined as follow:

$$
\begin{aligned}
& R_{T}=g \beta_{T} \Delta T^{\prime} K L^{\prime} / \alpha v \\
& N=\beta_{S} \Delta S^{\prime} / \beta_{T} \Delta T^{\prime} \\
& \begin{array}{l}
L e=\alpha / D \\
H a=B_{0} \sqrt{K \grave{o} / \mu} \\
\varepsilon=\varepsilon^{\prime} / \sigma
\end{array}
\end{aligned}
$$

The normalized porosity and the Lewis number are fixed at $\varepsilon=1$ and $L e=10$ in the present study. The heat and solute transfers across the cavity are given in terms of the Nusselt and Sherwood numbers defined as:

$$
\left.\begin{array}{c}
N u=\left.\int_{0}^{1} \frac{\partial T}{\partial Y}\right|_{Y=1} d X \\
S h=\left.\int_{0}^{1} \frac{\partial \mathrm{S}}{\partial \mathrm{X}}\right|_{\mathrm{X}=0} \mathrm{dY}
\end{array}\right\}
$$




\section{NUMERICAL MEHTOD}

The governing equations are discretized using a central finite-difference scheme combined with the alternate direction implicit method (ADI). Details concerning the validation of the present code in the absence of the magnetic field were reported by Bourich et al. (2004). Its validity in the presence of a magnetic field was proved in the present study by comparing our results with those of Altawalbeh et al. (2013) as illustrated in Table 1.

Table 1 Validation of the numerical code for $R_{T}=200, N=1, L e=10$ and various values of $\mathrm{Ha}$.

\begin{tabular}{|c|c|c|c|c|}
\hline & & $\Psi_{\max }$ & $\mathrm{Nu}$ & $S h$ \\
\hline \multirow{3}{*}{$H a=0$} & $\begin{array}{l}\text { Altawalbeh } \\
\text { et al. (2013) }\end{array}$ & 8.564 & 4.18 & 26.3 \\
\hline & $\begin{array}{l}\text { Present } \\
\text { study }\end{array}$ & 8.438 & 4.062 & 24.53 \\
\hline & $\begin{array}{l}\text { Deviation } \\
\text { (\%) }\end{array}$ & 1.47 & 2.82 & 6.73 \\
\hline \multirow{3}{*}{$H a=1$} & $\begin{array}{l}\text { Altawalbeh } \\
\text { et al. (2013) }\end{array}$ & 5.328 & 3.293 & 25.458 \\
\hline & $\begin{array}{l}\text { Present } \\
\text { study }\end{array}$ & 5.095 & 3.174 & 23.829 \\
\hline & $\begin{array}{l}\text { Deviation } \\
\text { (\%) }\end{array}$ & 4.37 & 3.61 & 6.4 \\
\hline \multirow{3}{*}{$H a=5$} & $\begin{array}{l}\text { Altawalbeh } \\
\text { et al. (2013) }\end{array}$ & 1.2 & 1.273 & 9.219 \\
\hline & $\begin{array}{l}\text { Present } \\
\text { study }\end{array}$ & 1.177 & 1.267 & 8.990 \\
\hline & $\begin{array}{l}\text { Deviation } \\
\text { (\%) }\end{array}$ & 1.9 & 0.47 & 2.5 \\
\hline
\end{tabular}

The comparison was performed in terms of $\Psi_{\max }$, $N u$ and $S h$ for $R_{T}=200, N=1, L e=10$ and three values of $H a(0,1$ and 5). The maximum deviation observed between our results and those of these authors is about $6.73 \%$. The validation of the numerical code is reinforced by presenting also qualitative comparisons in terms of contour lines for $R_{T}=200, H a=5, L e=1$ and $N=5$ (a) and $L e=10$ and $N=1$ (b). The comparative results presented in Figs. 2a-b show a good agreement between our results (on the right) and those of Altawalbeh et al. (2013) (on the left).

The effect of the grid size on the results corresponding to the monocellular, bicellular and tricellular flows obtained in this study was analyzed by using the uniform grids $61 \times 61,101 \times 101$ and $151 \times 151$. Tables 2 and 3 show that the characteristics of the flow and heat and mass transfer undergo negligible variations (lower than $0.4 \%$ ) when the grid $101 \times 101$ is replaced by a finer grid $151 \times 151$. However, when the grid $61 \times 61$ is replaced by the grid $101 \times 101$ the results of Tables 2 and 3 show a maximum deviation of about $11 \%$ for Sherwood number. Based on these results, a grid of $101 \times 101$ nodes was adopted in the present study.

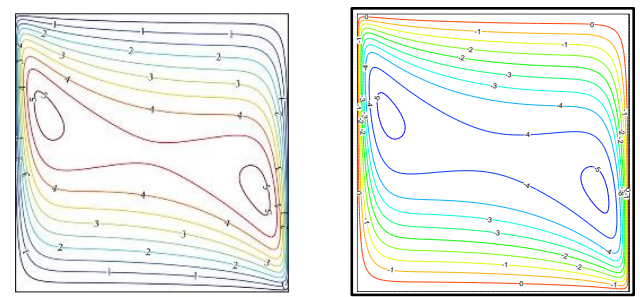

Streamlines

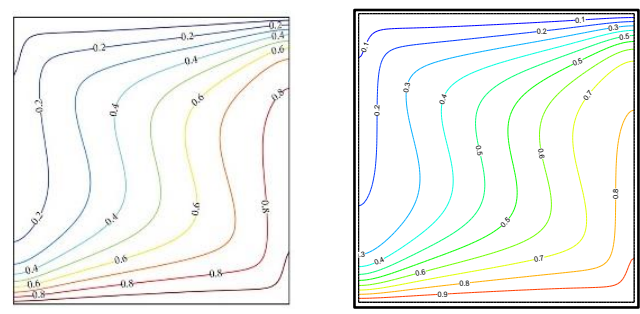

Isotherms

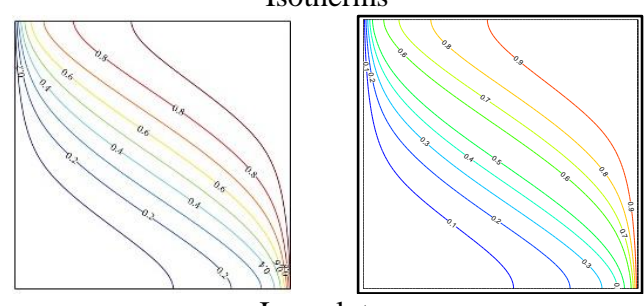

Iso-solutes

(a)
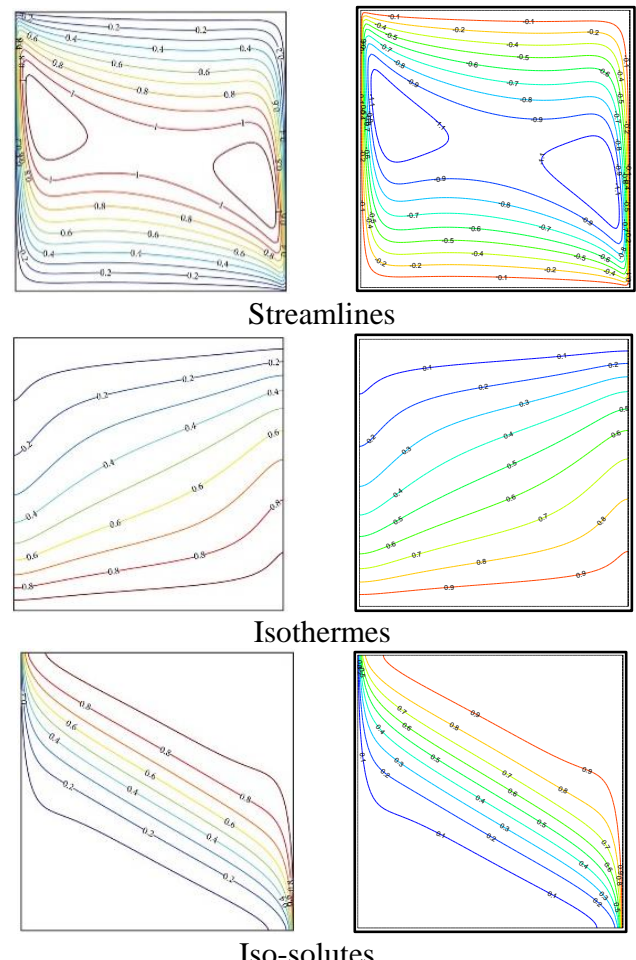

Altawalbeh et al. (2013)

Present study

(b)

Fig. 2. Comparison between our results and those of Altawalbeh et al. (2013) in terms of streamlines, isotherms and iso-solutes for $R_{T}=$ 200, $H a=5: L e=1$ and $N=5$ (a) and $L e=10$ and $N=1$ (b). 
Table 2 Effect of the Grid Size for $R_{T}=1000, L e=10, N=0$ and Various Values of $H a$.

\begin{tabular}{|c|c|c|c|c|c|}
\hline$H a$ & Flow & Grids & $\left|\Psi_{\text {ext }}\right|$ & $N u$ & $S h$ \\
\hline \multirow{3}{*}{0} & \multirow{3}{*}{$\mathrm{TF}$} & $61 \times 61$ & 14.205 & 9.258 & 8.863 \\
\cline { 3 - 6 } & & $101 \times 101$ & 13.93 & 9.151 & 9.966 \\
\hline & \multirow{3}{*}{3} & $151 \times 151$ & 13.927 & 9.146 & 9.974 \\
\cline { 3 - 6 } & \multirow{3}{*}{$\mathrm{BNF}$} & $61 \times 61$ & 8.099 & 4.666 & 10.223 \\
\cline { 3 - 6 } & & $101 \times 101$ & 8.110 & 4.648 & 10.679 \\
\cline { 3 - 6 } & \multirow{3}{*}{$\mathrm{TF}$} & $151 \times 151$ & 8.112 & 4.642 & 10.659 \\
\cline { 3 - 6 } & & $61 \times 61$ & 7.927 & 5.165 & 7.176 \\
\cline { 3 - 6 } & & $101 \times 101$ & 7.903 & 5.156 & 7.550 \\
\hline & & $151 \times 151$ & 7.896 & 5.152 & 7.554 \\
\hline
\end{tabular}

Table 3 Effect of the Grid Size for $R_{T}=200, L e=10, N=0.2$ and Various Values of $\mathrm{Ha}$.

\begin{tabular}{|c|c|c|c|c|c|}
\hline$H a$ & Flow & Grids & $\left|\Psi_{\text {ext }}\right|$ & $N u$ & $S h$ \\
\hline \multirow{3}{*}{0} & \multirow{3}{*}{ MTF } & $61 \times 61$ & 9.148 & 4.027 & 15.930 \\
\cline { 3 - 6 } & & $101 \times 101$ & 9.156 & 4.022 & 16.119 \\
\cline { 3 - 6 } 2 & \multirow{3}{*}{ BNF } & $151 \times 151$ & 9.159 & 4.020 & 16.056 \\
\hline & & $101 \times 61$ & 2.665 & 1.924 & 11.839 \\
\cline { 3 - 6 } & & $151 \times 151$ & 2.672 & 1.929 & 11.905 \\
\cline { 3 - 6 } & & & & 1.931 & 11.876 \\
\hline
\end{tabular}

\section{RESULTS AND DISCUTION}

The steady solutions submitted to the effect of a magnetic field in the present work are characterized by monocellular clockwise/trigonometric flow, bicellular natural/antinatural flow, and tricellular flow with a clockwise/trigonometric central cell and trigonometric/clockwise lateral cells, denoted $\mathrm{MCF} / \mathrm{MTF}, \mathrm{BNF} / \mathrm{BAF}$, and TCF/TTF, respectively. The streamlines (left), isotherms (middle) and isoconcentrations (right) corresponding to these solutions are illustrated in Figs. 3a-c for $R_{T}=200$, $L e=10, N=0$ and $H a=0$. Other details about these solutions are available in the work by Mansour et al. (2006). Note that, for $N=0$ (i.e. in the absence of solutal buoyancy forces), the intensities of the flow cells and the mean heat and mass transfer corresponding to $\mathrm{MCF}$, TTF, and BNF are identical to those corresponding to MTF, TCF, and BAF, respectively.

The effect of the magnetic field and the multiplicity of solutions on the variations of $N u$ and $S h$ vs. $R_{T}$ in the absence of solutal buoyancy forces is illustrated in Figs. 4a-c and Figs. 5a-c for $L e=10, N=0$ and $H a=0,1$ and 3 . Note that for $H a=0$, the monocellular (MF), bicelllular (BF) and tricellular (TF) flows were obtained for $R_{T} \geq 40, R_{T} \geq 80$ and $R_{T} \geq 160$, respectively. As expected, the increase of the Hartmann number retards the appearance of the different solutions. Hence, for $\mathrm{Ha}=1 /(\mathrm{Ha}=3)$ the monocellular, bicelllular and tricellular flows were obtained for $R_{T} \geq 59 /\left(R_{T} \geq 295\right), R_{T} \geq 88 /\left(R_{T} \geq 173\right)$ and $R_{T} \geq 197 /\left(R_{T} \geq 270\right)$, respectively. Furthermore,
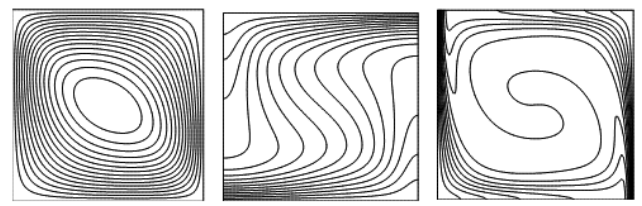

(a)
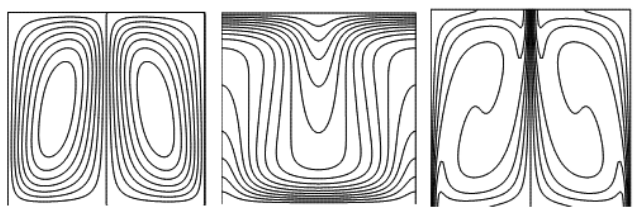

(b)
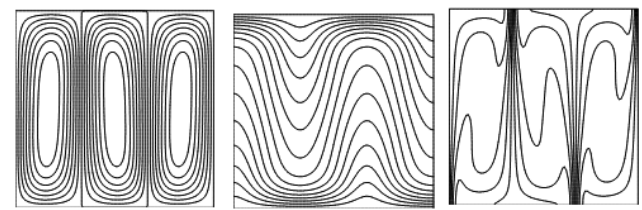

(c)

Fig. 3. Streamlines, isotherms and iso-solutes of monocellular flow (a), bicellular flow (b) and tricellular flow (c) obtained at $R_{T}=200, L e=10$, $N=\mathbf{0}$ and $H a=0$.

in the absence of the magnetic field, the transitions of the MF and $\mathrm{BF}$ toward oscillatory regimes occur at $R_{T}=382$ and 710, respectively. For $H a=1$, these transitions are registered at $R_{T}=445$ and 825 . By increasing the Hartmann value to 3 , the transition of the MF toward the oscillatory regime is delayed until 


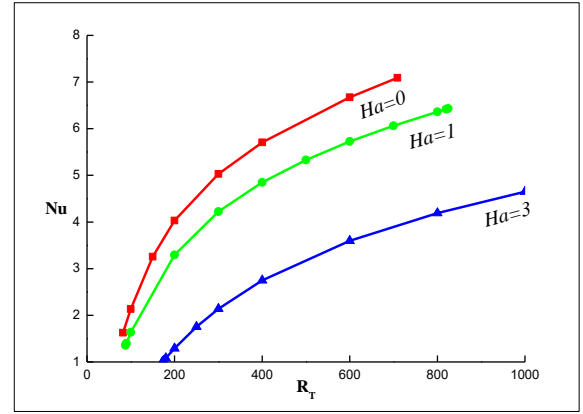

(a)

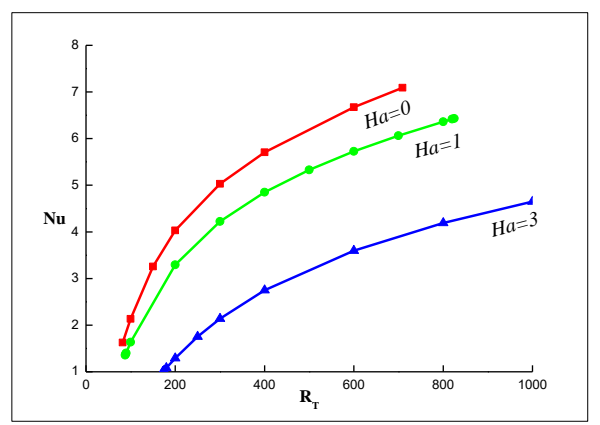

(b)

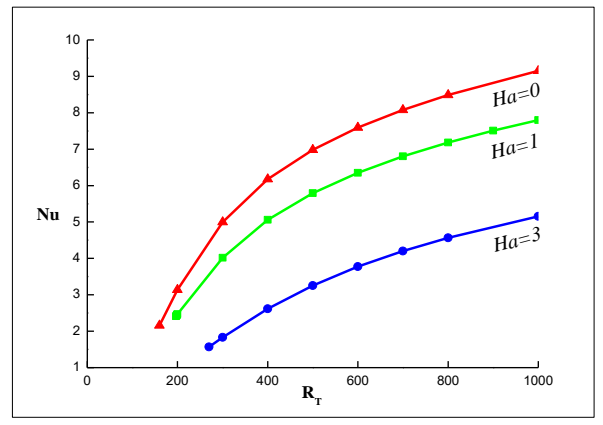

(c)

Fig. 4. Variations of $\mathrm{Nu}$ vs. $R_{T}$ for $L e=10, N=0$ and various values of $\mathrm{Ha}$ : (a) monocellular flow,

(b) bicellular flow and (c) tricellular flow.

$R_{T}=862$, while the BF remains steady even for $R_{T}$ exceeding 1000 . Figures $4-5$ show clearly that the increase of the magnetic field intensity reduces both heat and mass transfer rates. For example, at $R_{T}=300$, by increasing $\mathrm{Ha}$ from 0 to $3, \mathrm{Nu} / \mathrm{Sh}$ decreases by about $64.5 \% / 63.6 \%, 57.5 \% / 46.8 \%$ and $63.4 \% / 43.4 \%$ for the MF, the $\mathrm{BF}$ and the $\mathrm{TF}$, respectively.

The effect of the multiplicity of solutions on the generated heat and mass transfers is illustrated in Figs. 6a-c and Figs. 7a-c where the evolutions of $N u$ and $S h$ vs. $R_{T}$ are exemplified for the monocellular, bicelllular and tricellular flows in the cases of $H a=0,1$ and 3. The arrows in these figures indicate the transitions undergone by each solution when $R_{T}$ was progressively decreased starting from relatively large values of this parameter. It was observed that, for $H a=0$ and 1 , the TF transits towards the $\mathrm{BF}$ at $R_{T}=159$ and $R_{T}$ $=196$, respectively. The latter solution $(\mathrm{BF})$ transits toward the MF at $R_{T}=81$ and 87 for $H a=$ 0 and 1, respectively. As it can be seen from these figures, the above transitions are accompanied with an increase in heat and mass transfer. For $\mathrm{Ha}=3$, both the MF and TF transit toward the $\mathrm{BF}$ at $R_{T}=$ 294 and $R_{T}=268$, respectively. The transition of the TF toward the BF induces an enhancement of $\mathrm{Nu}$ and $\mathrm{Sh}$ while the transition from the MF toward the BF enhances $N u$ but reduces $S h$. In addition, the MF induces the weakest heat transfer in all its range of existence in the case of $\mathrm{Ha}=3$ (this is not the case for $H a=0$ and $H a=1$ ). Note also that, for $H a=0$ and 1 , the rest state is reached through the $\mathrm{MF}$ (when $R_{T}$ is progressively decreased), while for $H a=3$ the latter state is reached via the BF.

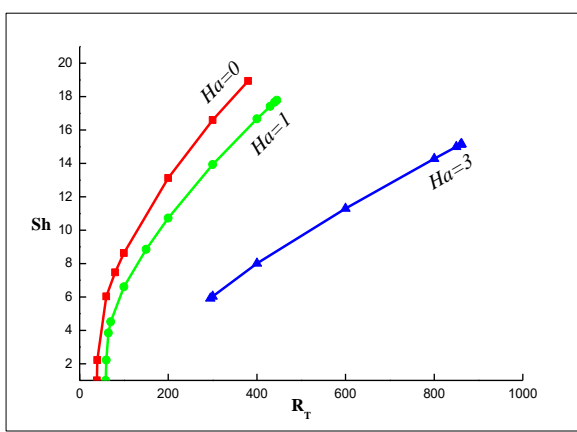

(a)

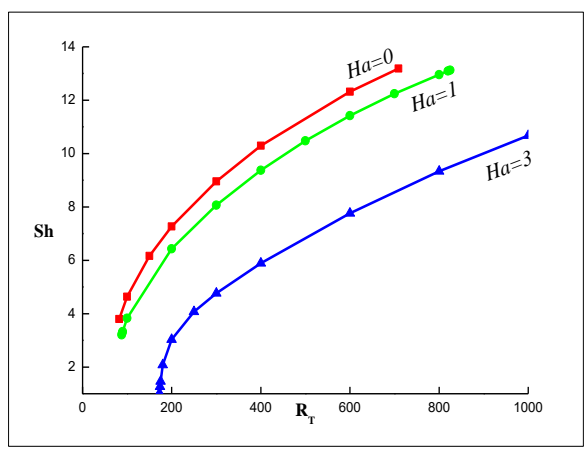

(b)

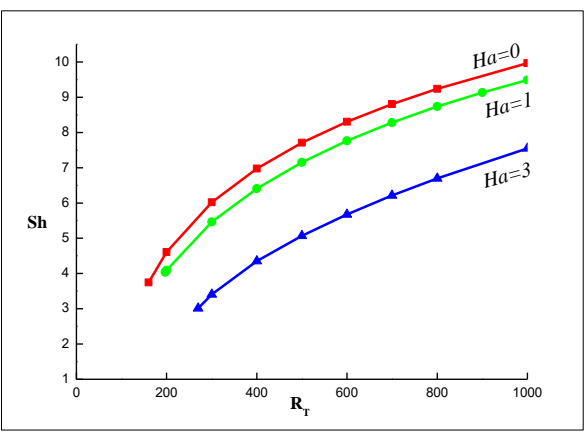

(c)

Fig. 5. Variations of Sh vs. $R_{T}$ for $L e=10, N=0$ and various values of $\mathrm{Ha}$ : (a) monocellular flow, (b) bicellular flow and (c) tricellular flow.

The effect of Hartmann number on the multiplicity of solutions and heat and mass transfer for $R_{T}=200$, 


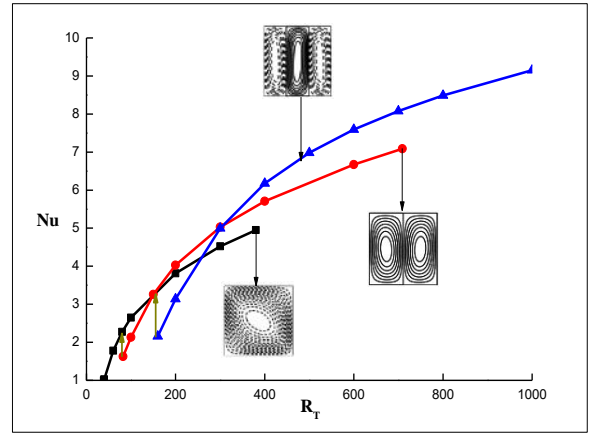

(a)

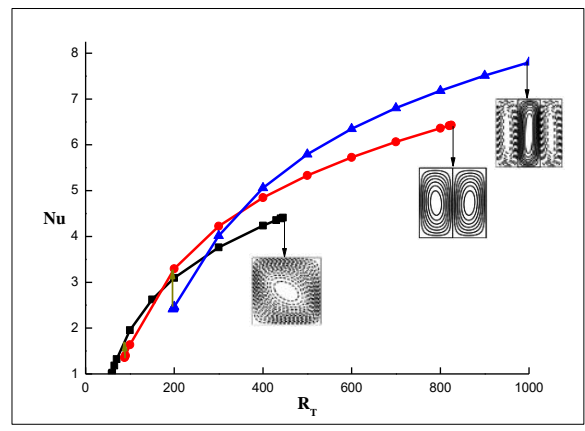

(b)

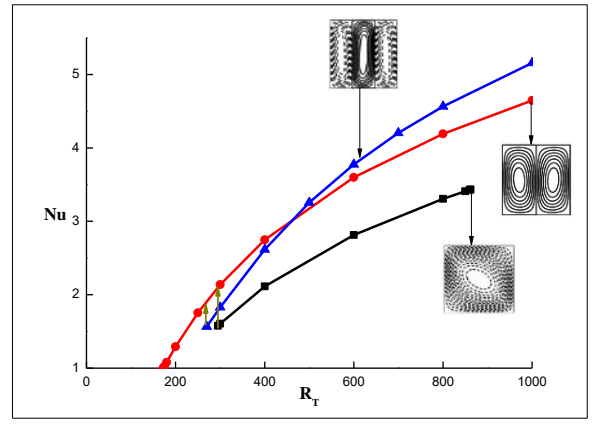

(c)

Fig. 6. Effect of $R_{T}$ on $N u$ corresponding to monocellular, bicellular and tricellular flows for $L e=10, N=0$ and $H a=0$ (a), 1 (b) and $3($ c).

$L e=10$ and $N=0$ is illustrated in Figs. 8a-b. These figures show that both the heat and mass transfer decrease by increasing the Hartmann number for all the flow modes considered. The arrows in these figures indicate the transitions obtained when $\mathrm{Ha}$ is progressively incremented from 0 . The critical values of $\mathrm{Ha}$ corresponding to these transitions are given in Table 4.

The progressive increase of $\mathrm{Ha}$ from 0 leads to the transitions of the TF and MF toward the $\mathrm{BF}$ at $H a_{c r}=1.067$ and $H a_{c r}=2.445$, respectively. The first transition is accompanied by an enhancement of about $31.6 \% / 55.1 \%$ for $\mathrm{Nu} / \mathrm{Sh}$ while the second transition leads to an increase of $N u$ by about $23.7 \%$ but to a decrease of $S h$ by about $18.9 \%$. However, the $\mathrm{BF}$ is maintained until the critical value $H a_{c r}=$ 3.35 , beyond which the flow vanishes and the heat and mass transfers are controlled by pure diffusion $(N u=S h=1)$. It should be mentioned that within the existence range of these three steady solutions, the $\mathrm{BF} / \mathrm{MF}$ induces the highest values of $\mathrm{Nu} / \mathrm{Sh}$, while the TF induces the weakest values for both $\mathrm{Nu}$ and $\mathrm{Sh}$.

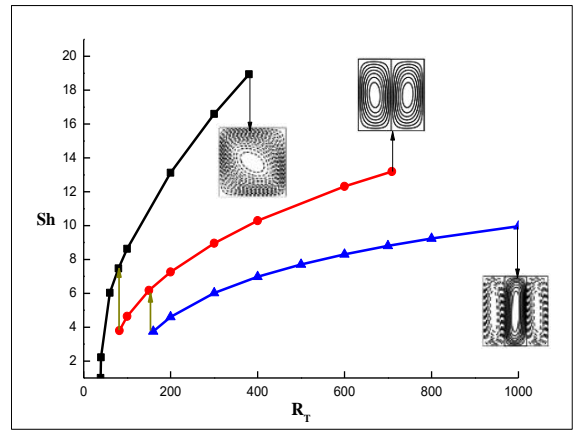

(a)

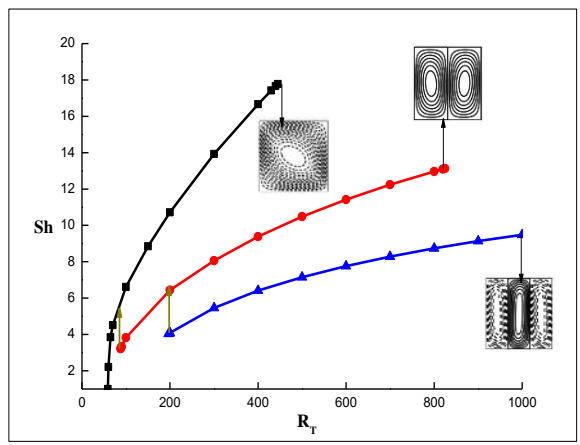

(b)

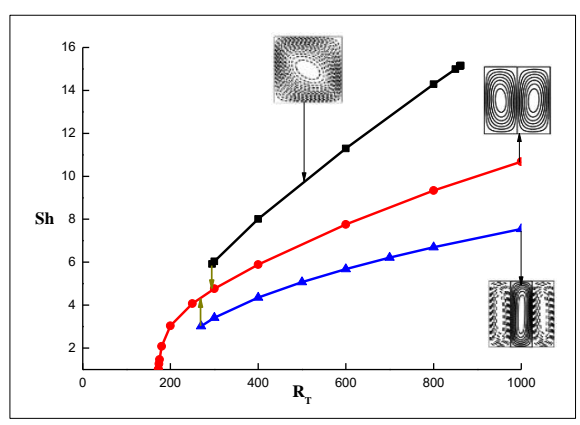

(c)

Fig. 7. Effect of $\boldsymbol{R}_{T}$ on $S h$ corresponding to monocellular, bicellular and tricellular flows for $L e=10, N=0$ and $H a=0$ (a), 1 (b) and 3 (c).

Table 4 Critical values of $\mathrm{Ha}$ corresponding to different transitions for $R_{T}=200, L e=10$ and $N=\mathbf{0}$.

\begin{tabular}{|l|c|c|c|c|}
\hline Flow mode & MCF & MTF & TCF & TTF \\
\hline $\begin{array}{l}\text { Transition } \\
\text { toward }\end{array}$ & BNF & BAF & BAF & BNF \\
\hline$H a_{c r}$ & 2.445 & 2.445 & 1.067 & 1.067 \\
\hline
\end{tabular}




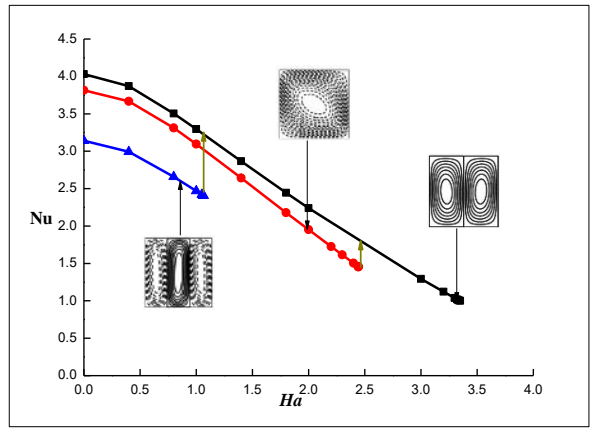

(a)

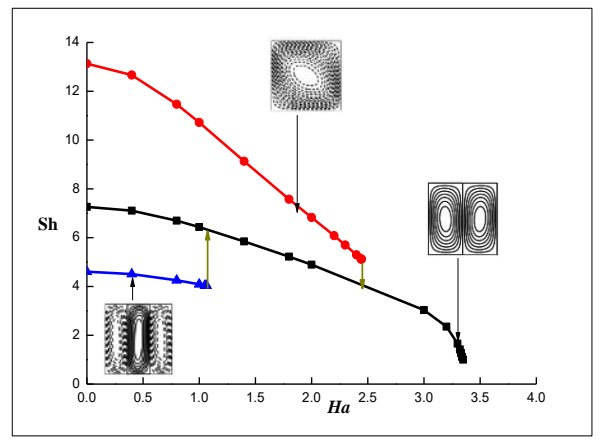

(b)

Fig. 8. Variations of $\mathrm{Nu}$ (a) and $S h$ (b) vs $\mathrm{Ha}$ for $R_{T}=200, L e=10, N=0$ and different flow modes.

The aim of this section is to emphasize the magnetic field effect on the multiple steady state solutions obtained in the presence of solutal buoyancy forces $(N \neq 0)$. Hence, Figs. 9-10 illustrate the evolutions vs. $N$ (varying in the range $-0.2<N<0.2$ ) of $N u$ and Sh corresponding to moncellular (MTF and MCF), bicellular (BNF and BAF) and tricellular (TTF and TCF) flows for $R_{T}=200, L e=10$ and $H a=0,1$ and 2 . It should be mentioned that the tricellular flows were not obtained for $H a=2$ and $R_{T}=200$. Note also that $N u$ and $S h$ corresponding to the MTF and TTF for $N>0$ are identical respectively to those induced by the MCF and TCF for $N<0$, whatever the Hartmann number value. In fact, the MTF/TTF and the MCF/TCF just exchange their roles while changing the sign of $N$. In addition, the BNF and the $\mathrm{BAF}$ induce identical average heat and mass transfers. Also, for the BF, the curves exemplifying the variations of $N u$ and $S h$ vs. $N$ are symmetrical with respect to the vertical line passing through $N=0$. Tables 5a-b summarize all the transitions observed and the corresponding critical values of $N$ leading to these transitions. It can be seen from Figs. 9-10 that the increase of $\mathrm{Ha}$ reduces strongly the range of $N$ corresponding to the tricellular mode. Moreover, Tables 5 indicate that the increase of $\mathrm{Ha}$ tends to reduce $N_{c r}$ (in absolute value) corresponding to the transitions towards the MCF for $N<0$ and the MTF for $N>0$.

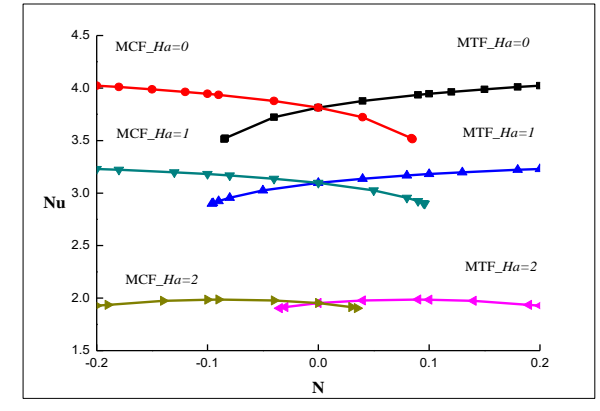

(a)

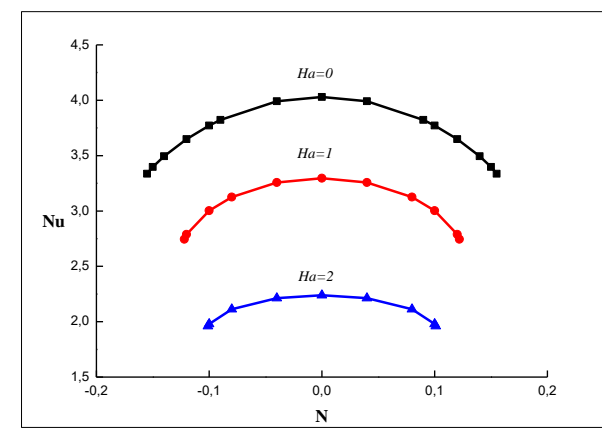

(b)

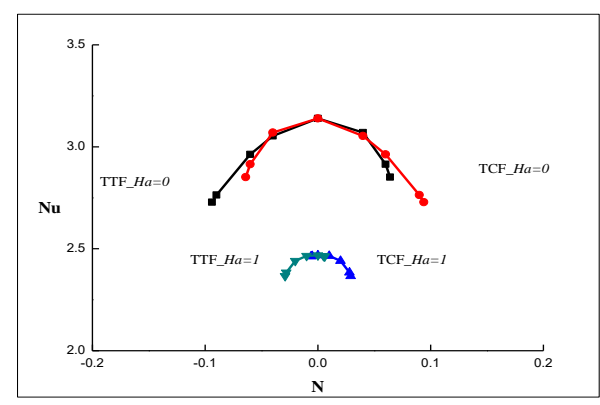

(c)

Fig. 9. Variations of $N u$ vs. $N$ for $R_{T}=200$, $L e=10$ and different values of $\mathrm{Ha}$ : (a) MTF and MCF, (b) BF and (c) TTF and TCF

\section{Conclusion}

The influence of the magnetic field on the multiplicity of solutions induced by thermosolutal convection in a square porous cavity submitted to a destabilizing vertical gradient of temperature and to a horizontal concentration gradient was considered in this work. The effect of the Hartmann number on the maintaining and disappearance of the multiple steady-state solutions obtained in the case of pure thermal convection is examined. Based on the obtained results, the following findings are deduced:

The presence of the magnetic field delays the transition towards the oscillatory regime when $R_{T}$ is increased. 


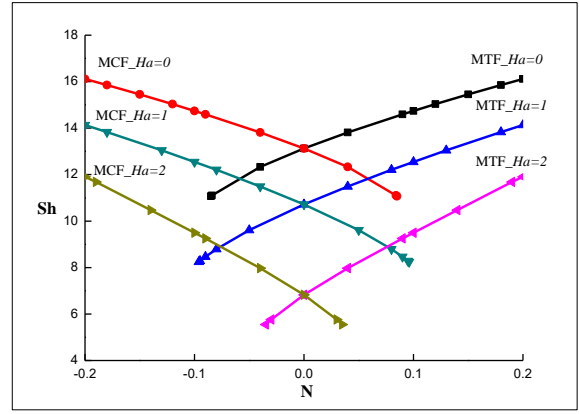

(a)

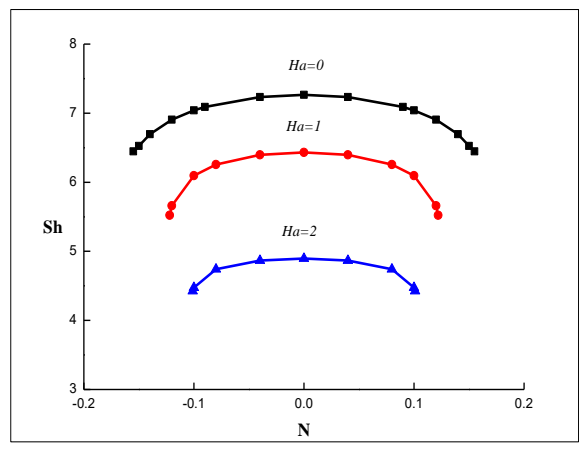

(b)

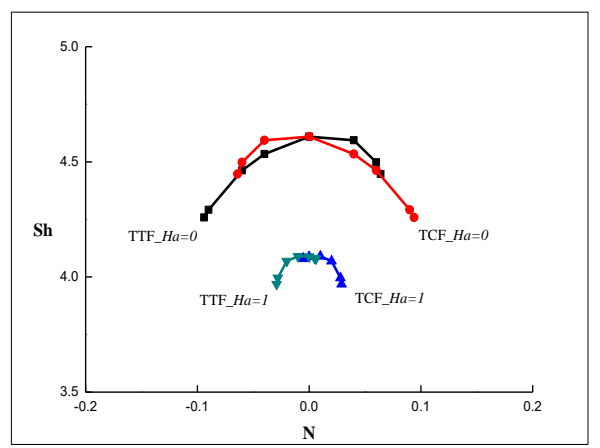

(c)

Fig. 10. Variations of $S h$ vs. $N$ for $R_{T}=200$, $L e=10$ and different values of $\mathrm{Ha}$ : (a) MTF and MCF, (b) BF and (c) TTF and TCF.

The increase of $H a$ rises the critical Rayleigh number above which the different flow modes exist. Hence, $R_{T_{-}}$rises from 40,80 and 160 to 295,173 and 270 when $H a$ is increased from 0 to 3 for the MF, BF and TF, respectively.

For $H a=0$ and 1 , the TF transits towards the $\mathrm{BF}$ and the latter transits toward the MF, while for $\mathrm{Ha}$ $=3$, both the MF and TF transit toward the BF, when $R_{T}$ is decreased starting from a value that allows the existence of the three solutions.

For $R_{T}=200, L e=10$ and $N=0$, the increase of $\mathrm{Ha}$ from 0 leads to the transitions of the TF and MF toward the $\mathrm{BF}$ at $H a_{c r}=1.067$ and $H a_{c r}=2.445$, respectively. The first transition is accompanied by an enhancement of $12.9 \%$ for $\mathrm{Nu}$ and $36.4 \%$ for $\mathrm{Sh}$, while the second transition leads to an increase of $\mathrm{Nu}$ by $25.1 \%$ but to a decrease of $\mathrm{Sh}$ by $54 \%$.

Tables 5 Transitions obtained for the different solutions and the corresponding critical values of $N$ for $R_{T}=200, L e=10$ and $H a=1,1$ and 2: (a) $N_{c r}>0$ and (b) $N_{c r}<0^{\circ}$.

(a)

\begin{tabular}{|c|l|l|l|}
\cline { 2 - 4 } \multicolumn{1}{c|}{} & $\begin{array}{l}\text { Flow } \\
\text { mode }\end{array}$ & $\begin{array}{l}\text { Transition } \\
\text { towards }\end{array}$ & $N_{c r}$ \\
\hline \multirow{5}{*}{$H a=0$} & MCF & BF & 0.086 \\
\cline { 2 - 4 } & BF & MTF & 0.156 \\
\cline { 2 - 4 } & TCF & MTF & 0.065 \\
\cline { 2 - 4 } & TTF & BF & 0.095 \\
\hline \multirow{5}{*}{$H a=1$} & MCF & BF & 0.097 \\
\cline { 2 - 4 } & BF & MTF & 0.123 \\
\cline { 2 - 4 } & TCF & MTF & 0.03 \\
\cline { 2 - 4 } & TTF & BF & 0.007 \\
\hline \multirow{2}{*}{$H a=2$} & MCF & BF & 0.036 \\
\cline { 2 - 4 } & BF & MTF & 0.102 \\
\hline
\end{tabular}

(b)

\begin{tabular}{|c|l|l|c|}
\cline { 2 - 4 } \multicolumn{1}{c|}{} & $\begin{array}{l}\text { Flow } \\
\text { mode }\end{array}$ & $\begin{array}{l}\text { Transition } \\
\text { towards }\end{array}$ & $N_{c r}$ \\
\hline \multirow{4}{*}{$H a=0$} & MTF & BF & -0.086 \\
\cline { 2 - 4 } & BF & MCF & -0.156 \\
\cline { 2 - 4 } & TCF & BF & -0.095 \\
\cline { 2 - 4 } & TTF & MCF & -0.065 \\
\hline \multirow{4}{*}{$H a=1$} & $\mathrm{MTF}$ & $\mathrm{BF}$ & -0.097 \\
\cline { 2 - 4 } & $\mathrm{BF}$ & $\mathrm{MCF}$ & -0.123 \\
\cline { 2 - 4 } & $\mathrm{TCF}$ & $\mathrm{BF}$ & -0.007 \\
\cline { 2 - 4 } & $\mathrm{TTF}$ & $\mathrm{MCF}$ & -0.03 \\
\hline \multirow{2}{*}{$H a=2$} & $\mathrm{MTF}$ & $\mathrm{BF}$ & -0.036 \\
\cline { 2 - 4 } & $\mathrm{BF}$ & $\mathrm{MCF}$ & -0.102 \\
\hline
\end{tabular}

Finally, by examining the combined effects of the buoyancy ratio, $\mathrm{N}$, and the Hartmann parameter, $\mathrm{Ha}$, on the existence of different flow modes we concluded that:

The critical values of $N$ corresponding to the transitions between the different solutions depend on the Hartmann number but the nature of the transitions remains unchanged.

The $N u$ and $S h$ corresponding to the MTF and TTF for $N>0$ are respectively identical to those induced by the MCF and TCF for $N<0$ regardless of the Hartmann number value. While the BNF and BAF induce identical average heat and mass transfers.

Generally, the increase of the magnetic field intensity reduces both heat and mass transfer rates for each flow mode.

\section{REFERENCES}

Ali, F. H., H. K. Hamzah, K. Egab, A. Müslüm and A. Shahsavar (2020). Non-Newtonian nanofluid natural convection in a U-shaped cavity under magnetic field. International Journal of Mechanical Sciences. 186, 105887. 
Altawalbeh, A., N. Saeid and I. Hashim (2013) Magnetic Field Effect on Natural Convection in a Porous Cavity Heating from below and Salting from Side. Hindawi Publishing Corporation Advances in Mechanical Engineering. Article ID 183079, 13 pages

Bahiraei, M. and M. Hangi (2015). Flow and heat transfer characteristics of magnetic nanofluids: A review. Journal of Magnetism and Magnetic Materials 374, 125-138.

Bourich, M., A. Amahmid and M. Hasnaoui (2004). Double diffusive convection in a porous enclosure submitted to cross gradients of temperature and concentration. Energy Conversion and Management 45, 1655-1670.

Chamkha, A. J. and H. Al-Naser (2002). Hydromagnetic double-diffusive convection in a rectangular enclosure with uniform side heat and mass fluxes and opposing temperature and concentration gradients. International Journal of Thermal Sciences 41, 936-948.

Chamkha, A. J. and H. Al-Naser (2002). Hydromagnetic double-diffusive convection in a rectangular enclosure with opposing temperature and concentration gradients. International Journal of Heat and Mass Transfer 45, 2465-2483.

Heidary, H., M. J. Kermani and M. Pirmohammadi (2016). Partition effect on thermo magnetic natural convection and entropy generation in inclined porous cavity. Journal of Applied Fluid Mechanics 9, 119-130.

Hoshyar, H. A., D. D. Ganji and A. R. Majidian (2016). Least square method for porous fin in the presence of uniform magnetic field. Journal of Applied Fluid Mechanics 9, 661-668.

Hussein, A., H. Ashorynejad, M. Shikholeslami and S. Sivasankaran (2014). Lattice Boltzmann simulation of natural convection heat transfer in an open enclosure filled with $\mathrm{Cu}$-water nanofluid in a presence of magnetic field. Nuclear Engineering and Design 268, 10-17.

Kabeel, A. E., Emad M. S. El-Said and S. A. Dafea (2015). A review of magnetic field effects on flow and heat transfer in liquids: Present status and future potential for studies and applications. Renewable and Sustainable Energy Reviews 25, 830-837.

Kasaeian, A., R. D. Azarian, O. Mahian, L. Kolsi, A. J. Chamkha, S. Wongwises and I. Pop g (2017). Nanofluid flow and heat transfer in porous media: A review of the latest developments. International Journal of Heat and Mass Transfer 107, 778-791.

Krakov, M. S. and I. V. Nikiforov (2002). To the influence of uniform magnetic field on thermomagnetic convection in square cavity.
Journal of Magnetism and Magnetic Materials 252, 209-211.

Maatki, C., L. Kolsi, H. F. Oztop, A. Chamkha, M. N. Borjini, H. Ben Aissia and K. Al-Salem (2013). Effects of magnetic field on 3D double diffusive convection in a cubic cavity filled with a binary mixture. International Communications in Heat and Mass Transfer 49, 86-95.

Mansour, A., A. Amahmid, M. Hasnaoui and M. Bourich (2006). Multiplicity of solutions induced by thermosolutal convection in a square porous cavity heated from below and submitted to horizontal concentration gradient in the presence of Soret effect. Numerical Heat Transfer Part A, 49, 69-94.

Nanjundappa, C. E., I. S. Shivakumara and H. N. Prakash (2012). Penetrative ferroconvection via internal heating in a saturated porous layer with constant heat flux at the lower boundary. Journal of Magnetism and Magnetic Materials 324, 1670-1678.

Ramambason Rakoto, D. S. and P. Vasseur (2007). Influence of a magnetic field on natural convection in a shallow porous enclosure saturated with a binary fluid. Acta Mechanical 191, 21-35.

Robillard, L., A. Bahloul and P. Vasseur (2006). Hydromagnetic natural convection of a binary fluid in a vertical porous enclosure. Chemical Engineering Communications 193, 1431-1444.

Sheikhnejad, Y., R. Hosseini and M. S. Avval (2017). Experimental study on heat transfer enhancement of laminar ferrofluid flow in horizontal tube partially filled porous media under fixed parallel magnet bars. Journal of Magnetism and Magnetic Materials 424, 16-25.

Sophy, T., H. Sadat and L. Gbahoue (2005). Convection thermomagnétique dans une cavité différentialement chauffée. International Communications in Heat and Mass Transfer 32, 923-930.

Teamah, M. A. and A. I. Shehata (2016). Magnetohydrodynamic double diffusive natural convection in trapezoidal cavities. Alexandria Engineering Journal 55, 1037-1046.

Teamah, M. A., A. F. Elsafty and E. Z. Massoud (2012). Numerical simulation of doublediffusive natural convective flow in an inclined rectangular enclosure in the presence of magnetic field and heat source. International Journal of Thermal Sciences 52, 161-175.

Zeng, M., Q. W. Wang, Z. P. Huang, G. Wang and H. Ozoe (2007). Numerical investigation of natural convection in an enclosure filled with porous medium under magnetic field. Numerical Heat Transfer, Part A 52, 959-971. 